\title{
Abortion care services delivered from a community sexual and reproductive health setting: views of health care professionals
}

\author{
Lucy Michie, ${ }^{1}$ Sharon T Cameron, ${ }^{2}$ Anna Glasier ${ }^{3}$
}

\begin{abstract}
${ }^{1}$ Clinical Research Fellow, Chalmers Sexual and Reproductive Health Centre, Edinburgh and Department of Reproductive and Developmental Sciences, University of Edinburgh, Edinburgh, UK ${ }^{2}$ Consultant Gynaecologist, Chalmers Sexual and Reproductive Health Centre, Edinburgh and Department of Reproductive and Developmental Sciences, University of Edinburgh, Edinburgh, UK ${ }^{3}$ Honorary Professor, Department of Reproductive and Developmental Sciences, University of Edinburgh, Edinburgh, UK
\end{abstract}

\section{Correspondence to Dr Lucy Michie, Chalmers Sexual and Reproductive Health Centre, 2a Chalmers Street, Edinburgh EH3 9E, UK; Lucy.Michie@ed.ac.uk}

Received 10 December 2012 Revised 13 March 2013 Accepted 20 March 2013 Published Online First 24 May 2013
To cite: Michie $\mathrm{L}_{\text {, }}$ Cameron ST, Glasier A. J Fam Plann Reprod Health Care 2013:39:270-275.

\begin{abstract}
Background Abortion services should provide high-quality contraceptive care. The community sexual and reproductive health (SRH) services may be well placed to deliver more abortion care in the UK. We wished to determine the views of health professionals working in SRH regarding their attitudes towards providing more abortion services and also the views of staff within one community SRH centre in Scotland where a service providing early medical abortion (EMA) was due to commence.
\end{abstract}

Methods An anonymous questionnaire distributed to attendees at a UK SRH scientific meeting collected data on demographics, current practice of and attitude to abortion, and views on delivery of abortion services. An internet questionnaire distributed by e-mail to staff at a community SRH clinic in Scotland sought demographics, views regarding the planned introduction of an EMA service and willingness to participate in it.

Results 165 questionnaires were completed out of 200 distributed at the scientific meeting (an $82 \%$ response rate). 128 (78\%) respondents felt that abortion services were suited to community clinics and 115 (70\%) stated that they would be willing to participate in them. $62 / 90$ (69\%) staff from the SRH clinic responded to the internet questionnaire. 44 (71\%) felt the plan to introduce abortion services was a natural extension to services already offered and the same number would be willing to be involved in such a service.

Conclusion There is clear support amongst health professionals in community SRH in the UK towards greater participation in the provision of abortion care services.

\section{INTRODUCTION}

Delivery of abortion care services throughout the UK is changing. In

\section{KEY MESSAGE POINTS}

- UK health professionals in sexual and reproductive health $(\mathrm{SRH})$ are generally supportive of providing abortion services from a community SRH setting.

- SRH staff consider abortion care to be a natural extension of existing services.

- Delivery of abortion services from a community SRH service would afford high-quality contraceptive provision and comprehensive management of sexually transmitted infections to women following abortion.

England and Wales in 2011, 61\% of all abortions were carried out in the independent sector, funded by the National Health Service (NHS), whilst 35\% were carried out in NHS hospitals. ${ }^{1}$ In contrast, in Scotland $98 \%$ of abortions are provided through the NHS and most of these are delivered from hospital-based departments of obstetrics and gynaecology. ${ }^{2} \mathrm{~A}$ key component of the care of women requesting an abortion, as directed in UK guidelines, is the provision of comprehensive counselling and immediate access following abortion to all available forms of contraception, in particular the longacting reversible methods. ${ }^{3}$ Indeed there is growing evidence that uptake of these effective methods of contraception, notably the intrauterine device (IUD) and intrauterine system (IUS) and the progestogen-only implant, is associated with a significantly reduced risk of repeat abortion. ${ }^{4-8}$ In some hospital settings the care of women requesting an abortion may be delegated to the more junior 
members of the medical staff, who often lack knowledge about contraception and the training to insert implants or intrauterine methods. While there is a lack of recent evidence regarding the attitudes of UK obstetrics and gynaecology trainees towards provision of abortion care, there are anecdotal reports that increasing numbers of them are choosing to opt out of abortion care for reasons of personal belief or because they find the work repetitive. A questionnaire of a proportion of both consultants and trainees in obstetrics and gynaecology in the UK in 1998 acknowledged similar concerns. The results identified that around one-third of trainees in obstetrics and gynaecology had not had any training in abortion procedures, a similar number stated a conscientious objection to abortion, and a number of consultants expressed views that some trainees also opted out of abortion for other reasons. ${ }^{9}$

It has been suggested that abortion services would be better provided in the community sexual and reproductive health (SRH) setting, since staff working within this area may be better placed to provide for women's ongoing contraceptive needs and have expertise in the insertion of IUDs and contraceptive implants. ${ }^{4}$ Additionally, SRH services may well be better for screening and testing for sexually transmitted infections (STIs) and may have more robust systems for partner notification.

Increasing numbers of women in Great Britain are undergoing early abortion, $78 \%$ of abortions in England and Wales having been performed at under 10 weeks' gestation and $65.5 \%$ in Scotland at under 9 weeks in 2011, ${ }^{1}{ }^{2}$ and increasing numbers of this group are opting for the medical method and choosing to go home soon after treatment to pass the pregnancy at home. ${ }^{1011}$ There is good evidence that early medical abortion (EMA) is highly amenable to delivery from a community setting and highly acceptable to women. ${ }^{12}$

The attitude of general practitioners, gynaecologists and medical students in the UK towards their involvement in provision of abortion has been the subject of previous research. ${ }^{13-16}$ However, no previous studies have focused on the views of those working within the field of SRH. In this study we aimed to determine the views of health professionals working in SRH regarding their attitudes towards a future role for specialists in SRH in providing more abortion care services by surveying delegates at the Annual Scientific Meeting of the Faculty of Sexual \& Reproductive Healthcare (FSRH) in the UK. In addition, we wished to determine the views of staff working within a community SRH centre in Edinburgh, Scotland, UK about the planned provision within the following 6 months of EMA within their integrated SRH service.

\section{METHODS}

To obtain the views and attitudes of a large number of health care workers, either working within or with an interest in SRH, we designed a questionnaire to distribute to all attendees at a large UK SRH scientific meeting (that of the FSRH) in April 2012. An introductory paragraph on the questionnaire explained its purpose and anonymity. Completed questionnaires were placed in sealed collection boxes. The questionnaire collected demographic data of the respondents including gender, age, current working role and geographical region of work, information on their current practice of and attitude to abortion, and their views on location of abortion care services. Responses in the sections relating to views on abortion and attitude and willingness to participate in, and location of, abortion care services were recorded by the participants on five-point Likert scales, the options ranging from 'strongly disagree' to 'strongly agree'. ${ }^{17}$

For the survey of staff within the community SRH service in Edinburgh, an anonymous internet questionnaire was distributed to all staff named on an up-to-date staff mailing list between January and March 2012. The questionnaire sought demographics including gender and role within the service, in addition to views regarding the planned introduction of the EMA service and willingness to participate in it. Responses consisted mostly of drop-down list options with additional freetext responses to selected questions.

\section{Statistics}

Data from both questionnaires were coded and entered onto separate databases using Microsoft Excel ${ }^{\mathrm{TM}}$. In the questionnaire of attendees at the SRH scientific meeting, responses relating to views on abortion, willingness to participate and location of services were combined such that 'strongly agree' and 'somewhat agree' were grouped as 'agree' whilst 'strongly disagree' and 'somewhat disagree' were grouped as 'disagree'. The remaining group of responses was 'neither agree nor disagree'. Data analysis was performed using IBM Statistical Package for Social Sciences (SPSS) software V.18 (IBM Corporation, New York, NY, USA). Groups were compared by Chi square $\left(\chi^{2}\right)$ test or Fisher's exact test, where counts within any individual cell of the contingency table fell below five. Statistical significance was deemed to be $p<0.05$.

\section{Ethical approval}

Both questionnaires were reviewed by the chair of a local research ethics committee who confirmed that ethical approval was not required as they constituted health services research.

\section{RESULTS}

A total of 165 questionnaires were returned out of 200 distributed at the UK SRH scientific meeting (an $82 \%$ response rate). Almost all respondents were female $(88 \%)$ and over two-thirds (73\%) were aged between 41 and 60 years. Over half of the respondents worked in England (54\%) and the majority 
were doctors (95\%). Demographics of the respondents are shown in Table 1 . Regarding any current involvement in abortion, only five (3\%) respondents stated they had no involvement in any aspect of abortion care; 129 (78\%) currently referred women for abortion, 106 (64\%) counselled and assessed women for consideration for abortion and 103 (62\%) signed the required legal paperwork for abortion. Only 24 (14\%) respondents stated that they either performed surgical abortion or administered the medications required for medical abortion.

Most respondents $(149 ; 90 \%)$ considered themselves to be 'broadly pro-choice'; six (4\%) were undecided and $10(6 \%)$ stated they were broadly antiabortion. There was no statistically significant difference between gender, age groups, region of work or current working role and personal view of abortion.

Statements were put forward to respondents regarding their view on location of abortion services, their willingness to participate, and views as to whether there is a role within SRH for abortion care. The responses are shown in Table 2. The majority of respondents $(128 ; 78 \%)$ attending the UK SRH

Table 1 Demographics of respondents to the questionnaire distributed at the Faculty of Sexual \& Reproductive Healthcare Annual Scientific Meeting in 2012

\begin{tabular}{|c|c|}
\hline Demographics & {$[n(\%)]$} \\
\hline \multicolumn{2}{|l|}{ Gender } \\
\hline Female & $146(88)$ \\
\hline Male & $18(11)$ \\
\hline Missing data & $1(1)$ \\
\hline \multicolumn{2}{|l|}{ Age range (years) } \\
\hline $20-30$ & $5(3)$ \\
\hline $31-40$ & $29(18)$ \\
\hline $41-50$ & $61(37)$ \\
\hline $51-60$ & $60(36)$ \\
\hline$>60$ & $10(6)$ \\
\hline \multicolumn{2}{|l|}{ Geographical region } \\
\hline Scotland & $65(39)$ \\
\hline England & $90(54)$ \\
\hline Wales & $3(2)$ \\
\hline England and Wales & $1(1)$ \\
\hline Northern Ireland & $2(1)$ \\
\hline Ireland & $1(1)$ \\
\hline Channel Islands & $3(2)$ \\
\hline \multicolumn{2}{|l|}{ Working role } \\
\hline Consultant & $35(21)$ \\
\hline General practitioner & $51(31)$ \\
\hline Staff grade/associate specialist grade doctor & $53(32)$ \\
\hline Trainee doctor & $16(10)$ \\
\hline Unspecified doctor & $2(1)$ \\
\hline Nurse & $6(3)$ \\
\hline No longer working & $1(1)$ \\
\hline Missing data & $1(1)$ \\
\hline
\end{tabular}

scientific meeting felt that abortion services were better suited to community clinics than hospital services. Eighty-three (50\%) respondents felt that services should be divided across community, hospital and non-NHS charitable and private organisations. Respondents working in England were statistically more likely to agree that abortion services were best suited to non-NHS charitable and private organisations compared to respondents working in other regions $(p=0.001)$. In addition, female respondents were statistically more likely to agree that abortion services were best suited to non-NHS charitable and private organisations compared to male respondents $(p=0.017)$. There were no other statistically significant differences in the responses to statements regarding location of services between gender, age groups, region of work or working role. Some 115 (70\%) respondents agreed that they would be willing in the future to participate in abortion services; 35 (21\%) disagreed while 13 (8\%) were undecided. The majority $(143 ; 87 \%)$ disagreed that there was no role in SRH for abortion services; eight (5\%) neither agreed nor disagreed and 11 (7\%) agreed there was no role. Significantly more women disagreed with this statement than men $(p=0.006)$. Respondents who considered themselves to be broadly anti-abortion were statistically more likely to disagree to participate in abortion services $(p=0.001)$ and statistically more likely to agree that there is no role in SRH for abortion services $(p=0.004)$.

The questionnaire of staff working within an SRH service in Edinburgh was distributed to 90 people, of whom $62(69 \%)$ responded. The majority $(56 ; 90 \%)$ of respondents were female. Twenty-four (39\%) respondents were nursing staff, $22(35 \%)$ doctors and $16(26 \%)$ administrative and clerical staff.

All were asked 'How do you feel about the plan for early medical abortion to take place in your service?' Forty-four (71\%) stated they felt this was a natural extension to the services already offered, four (6\%) were neutral and nine (15\%) were uncertain. Only five $(8 \%)$ respondents felt it was not an appropriate setting. There was no significant difference in responses to this question with gender and different working roles. In response to the question 'Would you be happy to be involved in such a clinic?', 44 (71\%) stated 'yes', seven (11\%) stated 'no' due to conscientious objection to abortion and $11(18 \%)$ were either uncertain or stated that this would not be of interest to them. Neither gender nor working role was associated with response to this question.

Respondents were asked, from a list of potential advantages, to select those that they felt would apply to women as a result of providing EMA within the service. Responses are shown in Table 3. One-third $(21 ; 34 \%)$ of respondents felt all were possible advantages to women.

Respondents were asked 'Do you feel there will be any potential disadvantages to women seeking an 


\begin{tabular}{|c|c|c|c|c|}
\hline \multirow[b]{2}{*}{ Statement } & \multicolumn{4}{|c|}{ Response [n (\%)] } \\
\hline & Agree & $\begin{array}{l}\text { Neither agree } \\
\text { nor disagree }\end{array}$ & Disagree & Missing \\
\hline Abortion services would be best suited to community clinics as opposed to a hospital setting & $128(78)$ & $24(14)$ & $12(7)$ & $1(1)$ \\
\hline Abortion services are best provided within a hospital-based setting in gynaecology & $31(19)$ & $44(27)$ & $88(53)$ & $2(1)$ \\
\hline Abortion services are best provided by separate non-NHS, private and/or charitable organisations & $18(11)$ & $53(32)$ & $93(56)$ & $1(1)$ \\
\hline Abortion services should be divided across these services & $83(50)$ & $49(30)$ & $29(18)$ & $4(2)$ \\
\hline $\begin{array}{l}\text { I would be willing to participate in abortion care for women, including relevant paperwork or } \\
\text { administering medication/undertaking procedure where appropriate }\end{array}$ & $115(70)$ & $13(8)$ & $35(21)$ & $2(1)$ \\
\hline I do not feel my role within SRH should have any involvement in abortion services & $11(7)$ & $8(5)$ & $143(87)$ & $3(2)$ \\
\hline
\end{tabular}

NHS, National Health Service; SRH, sexual and reproductive health.

abortion, in having their care delivered from the community SRH setting?' and were invited to specify what they considered the disadvantages to be. Thirty-two (52\%) felt there would be no disadvantages, $14(22 \%)$ thought there would and $16(26 \%)$ were uncertain. A total of eight possible disadvantages suggested by the respondents were: possible lack of anonymity within the community setting $(n=4)$; concern that the new abortion service would place undue additional workload on the existing services $(n=2)$; concern that some women may not wish to attend an SRH clinic for abortion due to possible stigma associated with sexual health clinics $(n=1)$; and that women may not wish to return to the SRH service in the future as it may remind them of having had an abortion $(n=1)$.

\section{DISCUSSION}

This study showed that most of the health professionals in SRH who were surveyed were generally supportive of providing abortion services from a community SRH setting. This is reassuring for future workforce provision of abortion services in the UK. Currently, those abortion services that are provided from hospital departments of obstetrics and gynaecology often compete for staffing with acute areas such as the labour ward, with the result that staffing of the abortion clinic may be delegated to junior, inexperienced members of the team. There is also evidence that trainees in obstetrics and gynaecology may lack interest in abortion management since a survey of

Table 3 Possible advantages for women of an abortion service located in Chalmers Sexual Health Centre, Edinburgh, UK

\begin{tabular}{ll}
\hline Possible advantages* & $\boldsymbol{n}(\%)$ \\
\hline Better provision of contraception post-procedure & $44(71)$ \\
More holistic approach to patient care & $42(68)$ \\
Opportunity to better manage sexually transmitted infections & $33(53)$ \\
More readily accessible site for patients & $32(52)$ \\
No response & $10(16)$ \\
\hline${ }^{*}$ Multiple advantages could be selected by each respondent. &
\end{tabular}

senior trainees in 2008 showed that only $2.8 \%$ had opted to undertake the advanced training module in abortion care. ${ }^{18}$ Our study confirms that doctors working within community SRH may be more willing to participate in abortion services. Additionally, abortion care is included as a mandatory module within the new training curriculum for UK specialist trainees in community SRH, ensuring that all doctors training in this new specialty gain knowledge and exposure of this integral part of SRH. ${ }^{19}$

Increasing numbers of women in Great Britain who request an abortion are at early gestation ( $\leq 9$ weeks), and increasing numbers are choosing to have an EMA method that enables them to leave the abortion service soon after treatment to pass the pregnancy at home. ${ }^{10} 11$ There is evidence that this method is highly amenable to provision in a community setting and that it is safe to do so, and furthermore it is acceptable to women. ${ }^{12}$ Our results show that the overwhelming majority of delegates at the SRH scientific meeting surveyed agreed that abortion services would be suited to a community SRH setting. Additionally, the majority of staff working in a community SRH clinic where abortion services were about to be introduced felt that this was a natural extension to the services already offered.

There are other reasons why it may be advantageous for more abortion care to be provided from community SRH clinics. First, it is possible that uptake of the most effective long-acting reversible methods of contraception (LARC) would be greater in a specialist contraceptive setting compared to a hospital setting, where hospital staff may lack specialist contraceptive knowledge or the ability to insert intrauterine contraception or progestogen-only implants. In our study, 7/10 staff surveyed at the community SRH clinic agreed that better contraceptive provision would be an advantage of providing abortion care through the SRH clinic. Immediate post-abortal provision of LARC is important as there is increasing evidence that insertion of an IUD/IUS or an implant is associated with a significantly reduced risk of having a further abortion. ${ }^{4-8}$ In a Scottish study of a hospitalbased abortion service, women who chose to have an 
IUD/IUS fitted were almost 18 times less likely, and women who chose to have a contraceptive implant inserted were 16 times less likely, to return for another abortion within the next 2 years, compared to those choosing to use an oral contraceptive pill. ${ }^{4}$

It would also seem only logical that an integrated community SRH service would also be better placed to manage STIs in women requesting abortion. Indeed, over half $(53 \%)$ of the respondents working within the community SRH clinic agreed that better management of STIs would be an advantage to offering abortion care within their setting. It has previously been shown that women who test positive for Chlamydia trachomatis at a hospital abortion service have poorer partner treatment rates than their counterparts who test positive at either a genitourinary medicine clinic or family planning clinics. ${ }^{20}$ This suggests that management of STIs amongst women requesting abortion may be particularly challenging for hospital services.

Of course it is possible that there may be some disadvantages to providing abortion care services from a community setting. Only a small number of staff from the SRH clinic reported possible disadvantages and these tended to be related to perceived increasing workload for themselves, or concerns that women may have less anonymity than in a hospital clinic. Clearly any abortion service must be able to provide guarantees of privacy and anonymity for women, and sexual health services are surely particularly sensitive to users' needs in this respect. A concern that was expressed by a minority of staff was that women might be reluctant to attend an SRH setting due to perceived stigma attached to a sexual health service. However, currently many women actually choose to attend SRH clinics to request a referral for abortion. Clearly, future qualitative research on the views and experiences of women attending abortion services in both hospital and community settings will be important to determine the location of services that women would consider most convenient and acceptable.

Although more than three-quarters of respondents from the scientific meeting felt that abortion services were suited to a community SRH setting, half also agreed that services should be divided across community, hospital and non-NHS organisations. Currently in England and Wales, abortion services are delivered from both the independent sector, funded by the NHS, and from NHS hospitals. This division of services has worked well for many years, although as suggested by the responses to our survey, these services could co-exist in both the independent sector and in an NHS community SRH setting. In Scotland the overwhelming majority of abortions are provided by hospitals, and while hospital services with surgical facilities and inpatient and day case beds will still be required, assessment clinics and facilities for EMA or early surgical abortion could also exist in community SRH clinics.

Clearly a potential drawback to our study is that most respondents from the scientific meeting were aged over 40 years, and so may not necessarily have reflected the views of younger health professionals or those still in training, who are the potential future providers of abortion services. There is currently a lack of recent qualitative research regarding attitudes towards abortion care of UK trainees in both obstetrics and gynaecology and SRH, and a future study in this area would be valuable. Nevertheless, our study showed that UK health professionals currently working in SRH are supportive of providing more abortion services in a community SRH setting. Clearly it will be important to evaluate service delivery from community SRH settings to determine if this model is indeed associated with the anticipated benefits for women and what, if any, the disadvantages may be.

Acknowledgements The authors are grateful to the Clinical Studies Group at the Faculty of Sexual \& Reproductive Healthcare for agreeing to the distribution of the study questionnaire during their session at the 2012 Faculty Annual Scientific Meeting.

Funding This project was undertaken with funding from Scottish Government, Public Health Division, as part of a series of projects aimed at improving the patient journey through abortion services.

Competing interests None.

Provenance and peer review Not commissioned; externally peer reviewed.

\section{REFERENCES}

1 Department of Health. Abortion Statistics, England and Wales: 2011. May 2012. http://www.wp.dh.gov.uk/transparency/files/ 2012/05/Commentary1.pdf [accessed 6 November 2012].

2 Information Services Division. Abortion Statistics 2011. May 2012. http://www.isdscotland.org/Health-Topics/Sexual-Health/ Publications/2012-05-29/2012-05-29-Abortions-Report-2011. pdf?55730837584 [accessed 6 November 2012].

3 Royal College of Obstetricians and Gynaecologists. The Care of Women Requesting Induced Abortion (Guideline No. 7). 2011. http://www.rcog.org.uk/files/rcog-corp/Abortion\%20guideline_ web_1.pdf [accessed 6 November 2012].

4 Cameron ST, Glasier A, Chen ZE, et al. Effect of contraception provided at termination of pregnancy and incidence of subsequent termination of pregnancy. Br J Obstet Gynaecol 2012;119:1074-1080.

5 Rose SB, Lawton BA. Impact of long-acting reversible contraception on return for repeat abortion. Am J Obstet Gynecol 2012;206:37.e1-e6.

6 Heikinheimo O, Gissler M, Suhonen S. Age, parity, history of repeat abortion and contraceptive choices affect the risk of repeat abortion. Contraception 2008;78:149-154.

7 Roberts H, Silva M, Xu S. Post abortion contraception and its effect on repeat abortions in Auckland, New Zealand. Contraception 2010;82:260-265.

8 Goodman S, Hendlish SK, Reeves MF, et al. Impact of immediate postabortal insertion of an intrauterine contraception on repeat abortion. Contraception 2008;78:143-148. 
9 Roe J, Francome C, Bush M. Recruitment and training of British Obstetrician-Gynaecologists for abortion provision: conscientious objection versus opting out. Reprod Health Matters 1999;14:97-105.

10 Cameron ST, Glasier A, Dewart H, et al. Women's experiences of the final stage of early medical abortion at home: results of a pilot survey. J Fam Plann Reprod Health Care 2010;36:213-216.

11 Lohr PA, Wade J, Riley L, et al. Women's opinions on the home management of early medical abortion in the UK. J Fam Plann Reprod Health Care 2010;36:21-25.

12 Ingham R, Lee E. Evaluation of Early Medical Abortion (EMA) Pilot Sites. London, UK: Department of Health, 2008.

13 Gleeson R, Forde E, Bates E, et al. Medical students' attitudes towards abortion: a UK study. J Med Ethics 2008;34:783-787.

14 Savage W, Francome C. British gynaecologists' attitudes in 2008 to the provision of legal abortion. J Obstet Gynaecol 2011;31:322-326.
15 Francome C, Savage W. Attitudes and practice of gynaecologists towards abortion in Northern Ireland. J Obstet Gynaecol 2011;31:50-53.

16 Finnie S, Foy R, Mather J. The pathway to induced abortion: women's experiences and general practitioner attitudes. J Fam Plann Reprod Health Care 2006;32:15-18.

17 Likert R. A technique for the measurement of attitudes. Arch Psychol 1932;140:1-55.

18 Royal College of Obstetricians and Gynaecologists. Survey of Training 2008. RCOG Training Committee 2008. http://www.rcog.org.uk/files/rcog-corp/uploaded-files/ RCOGSurveyof Training2008.pdf [accessed 7 November 2012].

19 Faculty of Sexual \& Reproductive Health. CSRH Module 3: Unplanned Pregnancy and Abortion Care. http://www.fsrh.org/ pdfs/SpecialtyModule3.pdf [accessed 27 November 2012].

20 Cameron ST, Glasier A, Scott G, et al. Novel interventions to reduce re-infection in women with chlamydia: a randomised controlled trial. Hum Reprod 2009;1:1-8. 